\title{
Experimental investigation of surface energy and subcritical crack growth in calcite
}

\author{
Anja Røyne, ${ }^{1}$ Jan Bisschop, ${ }^{2}$ and Dag Kristian Dysthe ${ }^{1}$ \\ Received 1 October 2010; revised 15 December 2010; accepted 10 January 2011; published 9 April 2011.
}

[1] Subcritical cracking behavior and surface energies are important factors in geological processes, as they control time-dependent brittle processes and the long-term stability of rocks. In this paper, we present experimental data on subcritical cracking in single calcite crystals exposed to glycol-water mixtures with varying water content. We find upper bounds for the surface energy of calcite that decrease with increasing water concentration and that are systematically lower than values obtained from molecular dynamics simulations. The relation of surface energy to water concentration can explain water weakening in chalks. The rate of subcritical crack growth in calcite is well described by a reaction rate model. The effect of increasing water on crack velocity is to lower the threshold energy release rate required for crack propagation. The slope of the crack velocity curve remains unaffected, something which strongly suggests that the mechanism for subcritical cracking in calcite does not depend on the water concentration.

Citation: Røyne, A., J. Bisschop, and D. K. Dysthe (2011), Experimental investigation of surface energy and subcritical crack growth in calcite, J. Geophys. Res., 116, B04204, doi:10.1029/2010JB008033.

\section{Introduction}

[2] Subcritical crack growth, refers to the phenomenon of slow crack propagation in brittle materials at stresses below the nominal failure stress [Lawn, 1993; Olagnon et al., 2006]. This phenomenon in of importance in the Earth's crust, where water and other active species are present and displacements and stresses are small. Subcritical cracking is argued to be the main mechanism of brittle creep of rocks [Heap et al., 2009; Scholz, 2002], and to control the time to failure at constant stress. Subcritical crack growth has thus been used as the underlying mechanism in models of slow earthquakes and aftershocks of major earthquakes [Helmstetter and Shaw, 2009].

[3] Calcitic rocks are abundant in many hydrocarbon bearing environments and other settings where reactive fluid transport is important. Faulting and fracturing of carbonate reservoirs have major implications for hydrocarbon fluid pathways [Agosta et al., 2007] and carbonates host many active seismic fault zones [Miller et al., 2004]. Experimental studies show that subcritical cracking plays an important role during the compaction of carbonate sediments [Croizé et al., 2010]. The physical processes controlling subcritical cracking in calcite are still, however, poorly known. Experiments on single calcite crystals [Dunning et al., 1994] have shown a nontrivial dependency on $\mathrm{pH}$ and ionic concentrations, while studies on calcitic rocks [Henry et al., 1977; Atkinson, 1984] have indicated that these rocks dis-

\footnotetext{
${ }^{1}$ Physics of Geological Processes, University of Oslo, Oslo, Norway.

${ }^{2}$ Institute for Building Materials, ETH Hönggerberg, Zurich, Switzerland.

Copyright 2011 by the American Geophysical Union. 0148-0227/11/2010JB008033
}

play a complex behavior, different to quartz bearing rocks and glasses. Various mechanisms including dissolution and microplasticity have been proposed to explain subcritical crack growth in calcite [Atkinson, 1984]. Unlike many other rocks, calcitic rocks display both plastic and brittle behavior at low stresses and temperature [Turner et al., 1954; Fredrich and Evans, 1989; Schubnel et al., 2006], and we therefore expect the fracture behavior of calcite to be more complex than the more commonly studied minerals such as mica and quartz.

[4] Chalk, a highly porous rock composed almost entirely of calcite, has been extensively studied due to its importance as an oil and gas producing reservoir rock. The mechanical strength of water saturated chalk is significantly lower than that of dry or oil saturated chalk. This so-called water weakening effect has been the subject of extensive study, but the underlying mechanism is still not fully understood. Risnes et al. [2005] showed that the strength of chalk decreases systematically with water content when saturated with water-glycol mixtures. Glycol is fully miscible with water, and its effect on chalk strength is similar to that of oil. Mixtures of water and glycol therefore allow for a systematic study of the effect of water concentration. Risnes et al. [2005] concluded that the water weakening effect is caused by the adhesion properties of water on the calcite surfaces.

[5] Brittle fracture propagation is controlled by the Griffith equilibrium condition, which can be stated as

$$
\mathcal{G}_{0}-2 \gamma_{s}^{e}=0,
$$

where $\mathcal{G}$ is the mechanical energy release rate with $\mathcal{G}_{0}$ corresponding particularly to the lower limit of crack propagation and $\gamma_{s}^{e}$ is the surface energy of the solid in contact with a given chemical environment. The presence of a chemically 


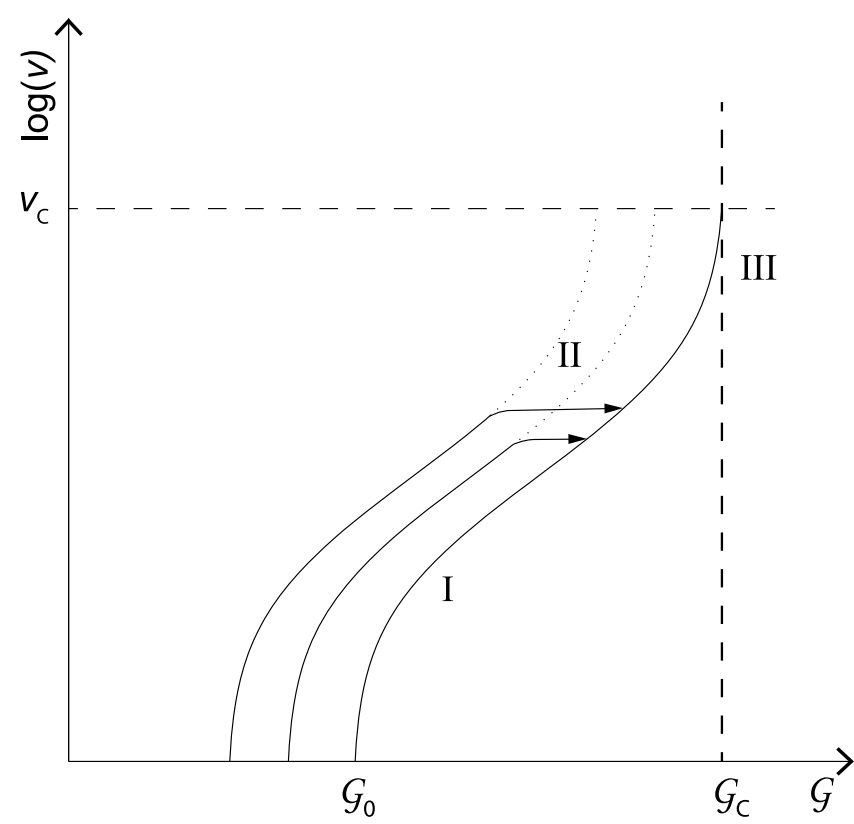

Figure 1. Schematic diagram of subcritical crack propagation [after Maugis, 1985]. The curve to the right corresponds to propagation in vacuum. The physical interpretation of regions I, II, and III is given in section 1.

active environment lowers the value of $\mathcal{G}_{0}$ and as a result the value of $v$ increases for a given value of $\mathcal{G}$ (Figure 1 ). Measured crack velocity curves $v(\mathcal{G})$ can generally be described by three regions of behavior. In region I, at low $\mathcal{G}$, crack propagation is controlled by reaction kinetics. When transport of active species to the crack tip becomes rate limiting, the system enters region II. The slope of $v(\mathcal{G})$ is typically much lower than in region I. Region III behavior takes place close to the critical energy release rate $\mathcal{G}_{c}$, where $v$ is a very strong function of $\mathcal{G}$.

[6] Subcritical crack propagation in region I (Figure 1) can be understood as a thermally activated process characterized by forward and backward energy barriers which are functions of $\mathcal{G}-2 \gamma_{s}^{e}$ [Vanel et al., 2009], and reaction rate theory gives the following relation for crack velocity $v[$ Wan et al., 1990a]:

$$
v=2 \nu_{0} a_{0} \exp \left(-\frac{\Delta F}{k T}\right) \sinh \left(\frac{\alpha\left(\mathcal{G}-2 \gamma_{s}^{e}\right)}{k T}\right),
$$

where $\nu_{0}=k T / h$ is a fundamental lattice vibration frequency, $k$ is the Boltzmann constant, $T$ is temperature, $a_{0}$ is some characteristic atomic spacing, $\Delta F$ is the quiescent value of the energy barrier, and $\alpha$ is an activation area. Experimental data from mica, sapphire and soda-lime silicate glass have been successfully fitted to this model [Wan et al., 1990a]. The phenomenological reaction rate theory does not specify the nature of the process involved.

[7] Surface energies of mineral-fluid interfaces control the presence of fluids at grain boundaries [de Gennes, 2003], create an upper bound for the stresses that can be generated by crystal growth in pores [Espinosa-Marzal and Scherer, 2010], and control mineral surface morphologies during growth and dissolution [de Leeuw and Parker, 1997].
Progress has been made in direct measurements of surface energies by advanced use of the surface forces apparatus [Alcantar et al., 2003; Anzalone et al., 2006] and recently also with atomic force microscopy [Hamilton et al., 2010], but most results from these methods are obtained on mica because of its atomically smooth surface. Molecular simulation studies have been used to determine the surface energies of calcite [de Leeuw and Parker, 1997; de Leeuw et al., 1998; de Leeuw and Cooper, 2004; Wright et al., 2001; Kerisit et al., 2003; Kvamme et al., 2009] with greatly varying results. Experimental measurements of the surface energy of calcite have been performed using fast fracture experiments with poor control on the environmental conditions [Gilman, 1960; Santhanam and Gupta, 1968]; surface energies have also been calculated from precipitation studies [Donnet et al., 2005, 2009]. As both of these methods are associated with large uncertainties, better experimental measurements of the surface energies of calcite are needed in order to test the numerical predictions. Subcritical fracture studies represent a complementary method for direct measurements of the surface energies of brittle materials in a range of chemical environments.

[8] In this paper, we present experimental results on the subcritical growth curves and surface energies of calcite in water-glycol mixtures with a range of water concentrations. The results are used to find upper bounds for the surface energies of calcite in these liquids, and to study the effect of water on the crack propagation velocities.

\section{Experimental Setup}

\subsection{Double Torsion Testing}

[9] We used the double torsion method [Evans, 1972] in our experiments (Figure 2). In this method, a flat sample with an initial notch or starting crack is loaded as shown in Figure 3. Bending of the sample results in propagation of a

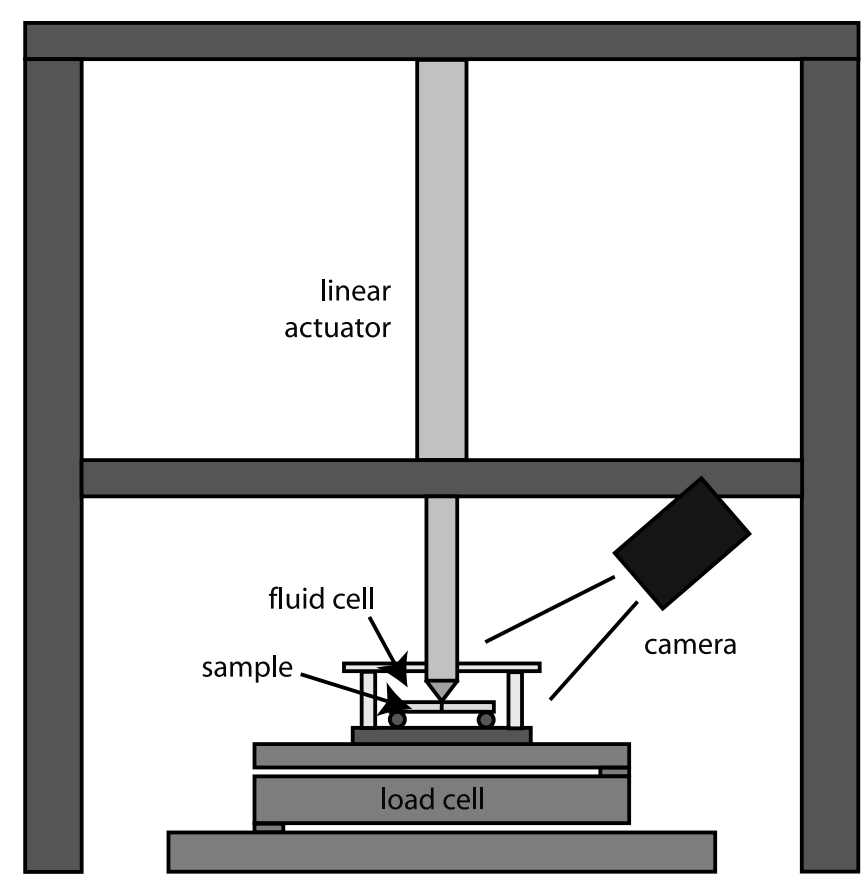

Figure 2. Schematic of the double torsion rig. 


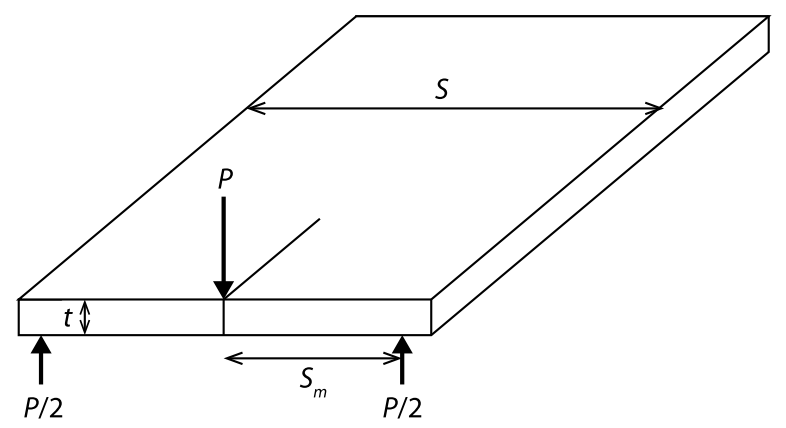

Figure 3. Loading of sample and sample dimensions in the double torsion method.

mode I crack with the highest tensile stress at the bottom side of the sample. This method is well suited for stable crack propagation studies. The energy release rate can be considered to be independent of crack length for the middle portion of the sample, where it can be calculated as [Shyam and Lara-Curzio, 2006]

$$
\mathcal{G}=\frac{3 P^{2} S_{m}^{2}}{2 S t^{4} G \psi}
$$

where $P$ is the applied load, $S_{m}$ is half the distance between the supports, $S$ and $t$ are sample width and thickness, $\psi=1-$ $0.6302 \tau+1.20 \tau \exp (-\pi / \tau)$ is a geometric correction factor with $\tau=2 t / S$, and $G$ is the shear modulus for calcite (32.8 GPa [Chen et al., 2001]).

[10] Equation (2) is derived from the analytical expression for the sample compliance $C$ [Shyam and Lara-Curzio, 2006],

$$
C=\frac{\Delta}{P} \approx \frac{3 S_{m}^{2}}{S t^{3} G \psi} a,
$$

where $\Delta$ is the displacement of the loading point. Experimentally, the compliance of the specimen is found to follow the relationship

$$
C=\frac{\Delta}{P}=B a+D,
$$

where $B$ and $D$ are scaling constants. The linear dependence of compliance on crack length is only observed in the middle part of the sample, where edge effects are negligible. Reliable measurements can therefore be made for the region of crack lengths where $C(a)$ is linear. The parameter $D$ reflects the compliance of the loading system, while $B$ should be equal to the prefactor in equation (3).

[11] The load relaxation method, where displacement of the loading point $\Delta$ is increased quickly and then left constant while the decay of the load $P$ is recorded, is perhaps the most commonly used method in double torsion tests. When the $\Delta$ is constant, then the crack velocity $v=\mathrm{d} a / \mathrm{d} t$ can be calculated from the relation

$$
v=\frac{-P_{i}}{P}\left(a_{i}+\frac{D}{B}\right) \frac{\mathrm{d} P}{\mathrm{~d} t},
$$

where $P_{i}$ and $a_{i}$ are instantaneous measurements of load and crack length. Ideally, $D / B \ll a$ and can be ignored so that only one crack length measurement is needed. This is particularly useful for nontransparent materials where identification of the crack tip is difficult. In measurements on transparent materials, calculation of $v$ from equation (5) is complementary to direct measurement of crack velocity from the recorded crack length $a$ as a function of time.

\subsection{Sample Preparation and Loading}

[12] Calcite samples measuring $30 \times 10 \times 1 \mathrm{~mm}$ were cut with the $10 \times 30 \mathrm{~mm}$ face parallel to the $\{10 \overline{1} 4\}$ cleavage plane. All of the other faces were cut normal to the $\{10 \overline{1} 4\}$ plane and thus miscut with respect to the calcite rhomb (Figure 4). The samples were prepared by Photox Optical Systems Ltd. from mined calcite crystals. The $10 \times 30 \mathrm{~mm}$ surfaces were optically polished. No heterogeneities were visible in the samples when examined between crossed polarizers.

[13] A starting crack was made by scratching near the edge of the sample to produce a slightly rough cleavage crack, $4-5 \mathrm{~mm}$ in length. The sample was then loaded in the rig and the crack was forced to propagate to the middle portion of the sample before measurements commenced. In other materials, a guide groove is often necessary to avoid significant crack deflection, and the results can sometimes be affected by the shape of the groove [Shyam and LaraCurzio, 2006], but this was not necessary in our samples due to the strong cleavage of calcite. Crack propagation in other crystallographic directions was not attempted as it is extremely difficult to fracture a single calcite crystal at an angle to a cleavage plane.

[14] All of the experiments were performed at room temperature, which varied with $\pm 0.5^{\circ} \mathrm{C}$ around $22^{\circ} \mathrm{C}$. The water concentration was varied using a mixture of distilled water in ethylene glycol, following the idea from Risnes et al. [2005]. The solubility of calcite in pure glycol is $30 \%$ lower than in pure water [Sandengen, 2006] and both fluid-air surface tension and dielectric constant of glycol are half those of water [Lide, 2008]. Several experiments were performed at each mole fraction of $0,0.1,0.3,0.5,0.7$ and 1 of water in glycol. Distilled water was used in all mixtures. The pure glycol may contain as much as $3 \%$ water as reported by the manufacturer. Glycol from the same bottle was used for all experiments.

\subsection{Experimental Procedure}

[15] Due to the small size of the samples (which was limited by the availability of high-quality crystals), the stiffness of the rig $D$ was high compared with the sample compliance $B$ (theoretical value $1.67 \times 10^{-4} \mathrm{~N}^{-1}$, measured values $B=2.5 \pm 0.4 \times 10^{-4} \mathrm{~N}^{-1}$ and $D=1.3 \pm 0.3 \times$ $10^{-5} \mathrm{~N}^{-1} \mathrm{~m}$ ). When the sample stiffness is comparable to the stiffness of the loading rig, only a narrow range of crack velocities can be measured in a single load relaxation experiment because $P$ decays less with $a$ than in a stiffer rig. In order to obtain data for as wide a range of stresses as possible, we used a combination of load relaxation measurements (Figure 5) and measurements where the displacement of the loading point was continuously increased. The rate of loading point displacement was increased from $10^{-9} \mathrm{~m} / \mathrm{s}$ at small crack velocities to $10^{-6} \mathrm{~m} / \mathrm{s}$ at high velocities. The crack velocities were calculated from the local derivatives of crack length and/or load relaxation 


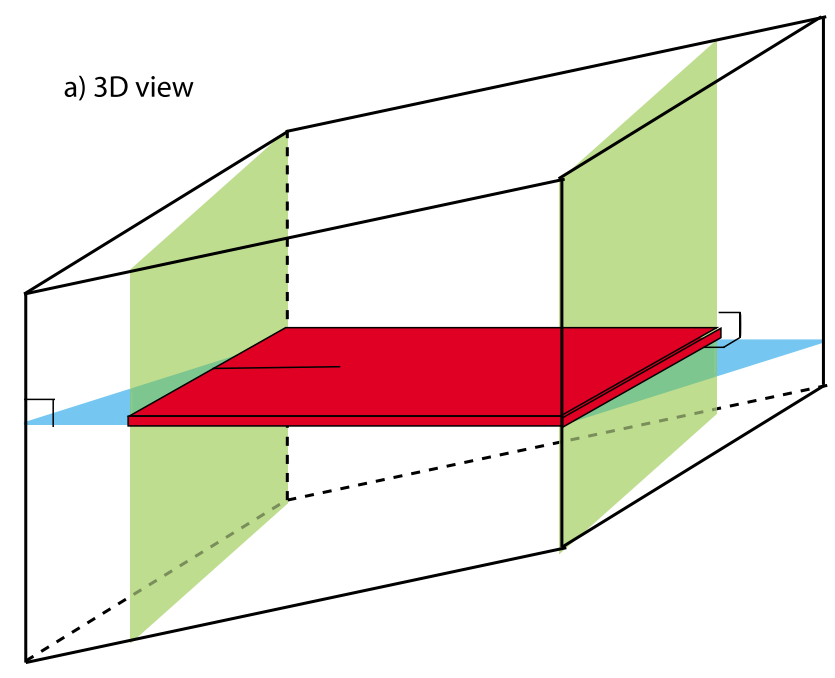

b) top view

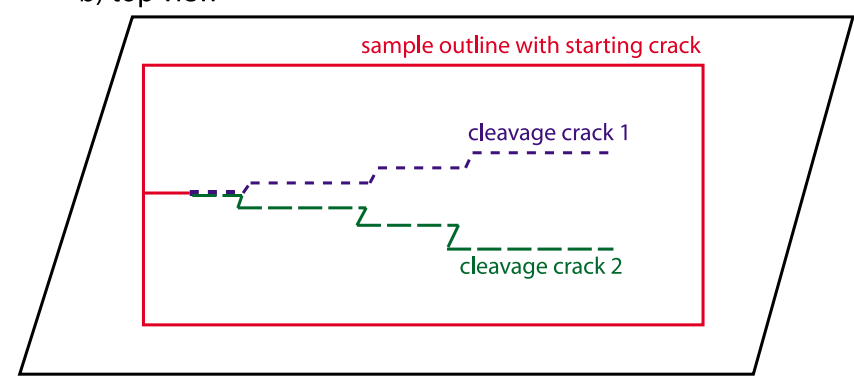

c) side view

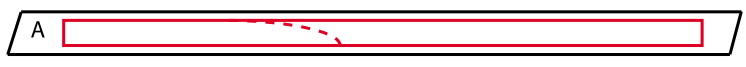

$\mathrm{B} \square$

Figure 4. Crystallographic orientation of sample. (a) Sample orientation in relation to the calcite rhomb. (b) Top view of sample, showing the two possible crack directions. The deviation from the center line is greatly exaggerated for clarity. The direction of crack 1 is expected to be more energetically favorable than crack 2. (c) Side view showing the two possible sample orientations. The angle between the cleavage plane and the crack front is larger in A than in $\mathrm{B}$, which may facilitate crack propagation in samples with the $\mathrm{B}$ orientation.

measurements (equation (5)) and the energy release rate $\mathcal{G}$ was found from instantaneous load measurements using equation (2).

\subsection{Data Acquisition, Processing, and Uncertainty Analysis}

[16] The position of the loading point was controlled by a linear actuator (PI M-227.50). The load $P$ was recorded by an Omega load cell with a maximum range of $40 \mathrm{~N}$, and read with a Keithley 2002 multimeter, giving a sensitivity in the load readings of about $\pm 0.001 \mathrm{~N}$. The crack was imaged by adjusting the light source to get maximum reflection from the crack surface, and the crack tip was identified automatically using Matlab software by plotting the light intensity along a profile parallel to the crack and recording where a threshold light intensity was exceeded (Figure 6). Since calcite is a transparent material, crack length measurements are much less problematic than in nontransparent and disordered materials. The linear relationship between compliance and crack length confirms that the crack length detection algorithm is consistent for the entire sample length. Pictures were obtained with a resolution of about $7.5 \mu \mathrm{m} / \mathrm{pixel}$ at a maximum rate of $3 \mathrm{~Hz}$.

[17] After each experiment, the crack surface was examined using a white light interferometer (Wyko NT1100 from Veeco). Scans over the entire crack surface were made with a vertical resolution of about $20 \mathrm{~nm}$. More detailed scans were also made of areas of size $120 \times 90 \mu \mathrm{m}$ with a vertical resolution of about $2 \mathrm{~nm}$. Smaller features, approaching the unit step height of calcite ( $5 \AA)$ were also visible in these scans, but not quantifiable.

[18] The crack velocities were found by measuring the derivative of crack length with respect to time, and also using equation (5) during load relaxation measurements. The derivatives were estimated by fitting a straight line to a suitable number of data points. This method resulted in an uncertainty in the measured velocity of up to a factor two, depending on the number and quality of pictures or load measurements in the relevant range. The uncertainty in $\mathcal{G}$ calculated from equation (2) is due mainly to the uncertainty in sample thickness $t(3 \%)$ and shear modulus $G(5-10 \%)$ and absolute position of the supports $S_{m}(5 \%)$ and is estimated to be about $15 \%$. The discrepancy between measured and theoretical sample compliance $B$ may reflect a systematic error leading to a systematic overestimation of $\mathcal{G}$.

[19] The rig was tested for relaxation several times before and between measurements, by leaving it without a sample or with a dummy sample made of stainless steel, at loads higher than those used in the experiments. The relaxation measured in the rig was negligible compared to that measured due to crack growth. In order to test repeatability, we performed some experiments on $76 \times 26 \times 1 \mathrm{~mm}$ glass
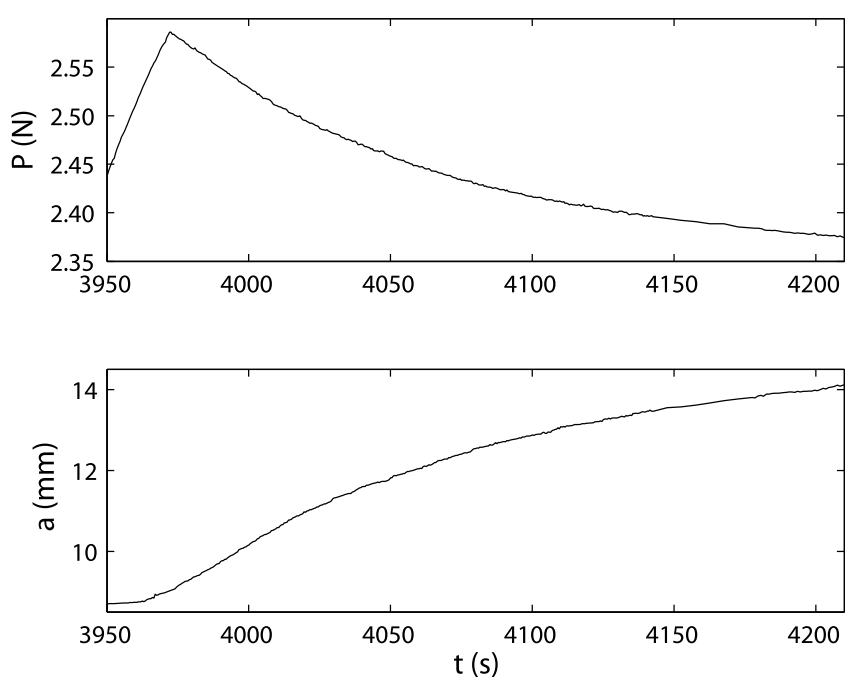

Figure 5. Load $P$ (Newtons) and crack length $a$ (millimeters) measured during one load relaxation experiment. The velocities measured from this plot are the upper portion of measurements shown in blue at $c_{w}=0.7$ in Figure 7. 

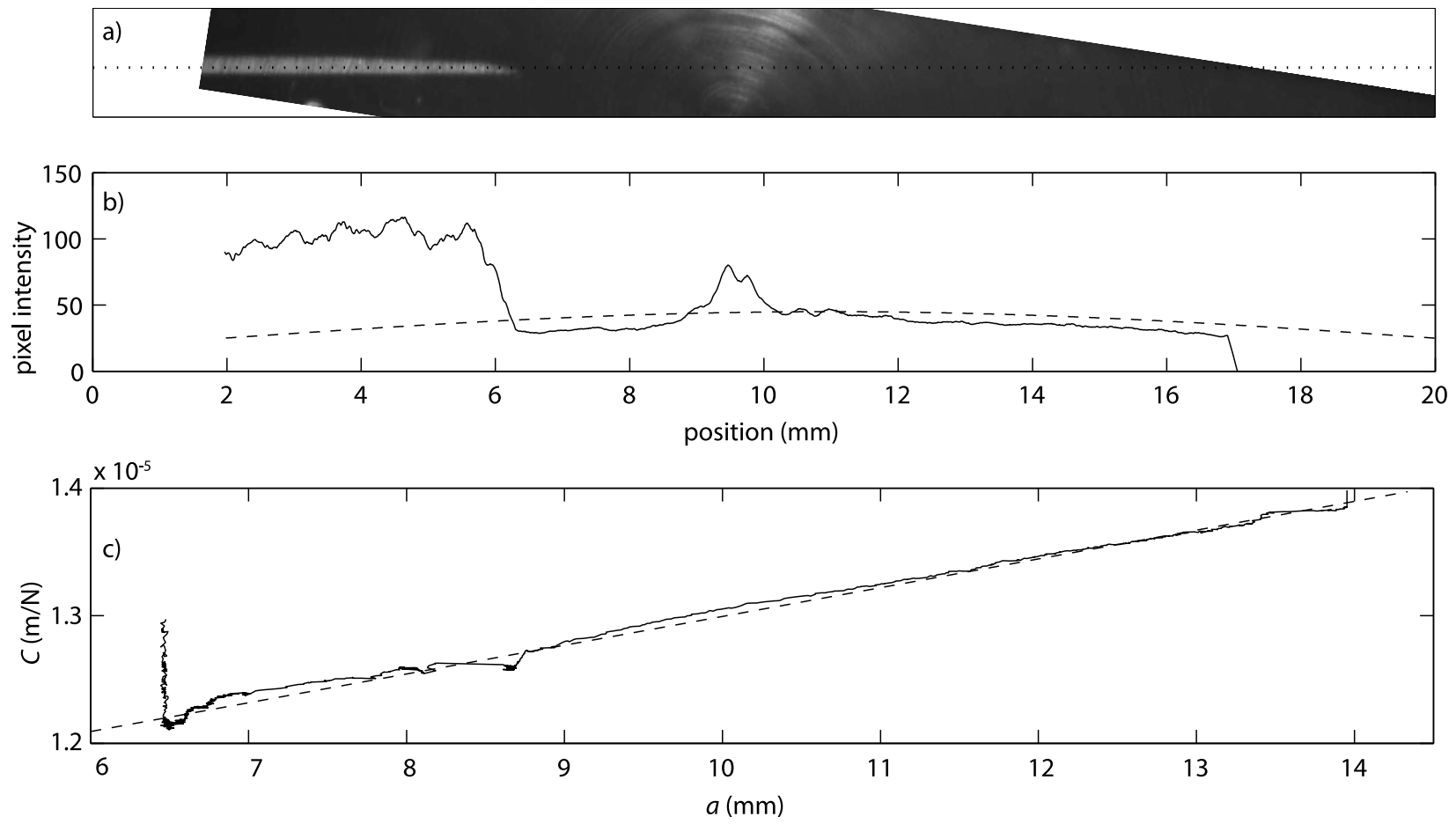

Figure 6. (a) Instantaneous image of the crack tip. The image was rotated to have the crack propagate along the $x$ axis of the picture. (b) Plot along the dotted line in Figure 6a, together with a threshold function (dashed line) which reflects the background intensity. The crack position was taken to be the point where the intensity crossed the threshold curve. When the crack tip was near locations in the cell with high reflections (seen at $10-11 \mathrm{~mm}$ in the plot), this had to be corrected manually, and some images were discarded. (c) Compliance curve for this sample. The dashed line shows a linear fit, $C=2.26 \times 10^{-4} a+$ $1.07 \times 10^{-5}$ (with $a$ in millimeters)

microscope slides with a very sharp starting crack and without a guide groove, loaded in the same way as the calcite samples. The measured $v(\mathcal{G})$ curves for the glass samples showed a high degree of reproducibility, much more than what we experienced for the calcite samples (see section 4).

\section{Results}

\subsection{Crack Velocities}

[20] The measured crack velocity $v$ as a function of energy release rate $\mathcal{G}$ from all our experiments are shown in Figure 7. Measured crack velocities range from $10^{-8}$ to $10^{-2} \mathrm{~m} / \mathrm{s}$. It can be seen that the crack velocity at a given $\mathcal{G}$ is increased by several orders of magnitude when the water concentration is raised.

[21] We find a significant variation in the shapes of the $v(\mathcal{G})$ curves that corresponds to variations in velocity from a factor 2 (pure water) to almost 3 orders of magnitude (pure glycol). This variability is found to be independent of loading method, sample alignment, crack deflection and crack roughness (see Figure 9) and strongly contrast the reproducibility of the tests we made on glass samples. The variation is of similar magnitude for experiments performed both on a single sample and for results for different samples. For any material, a higher energy release rate should always correspond to a higher crack velocity. However, we observe situations in a number of our experiments where $v$ decreases with $\mathcal{G}$ (see section 4).

[22] In the purely brittle regime all of the mechanical energy is converted to crack extension. It follows that the maximum velocity observed at any given $\mathcal{G}$ is the velocity closest to the true brittle $v(\mathcal{G})$ curve, while lower velocities are caused by additional dissipative processes. For each water fraction we have picked a number of maximum velocities (open black symbols in Figure 7) which we take to represent the "brittle $v(\mathcal{G})$ curve" for the respective conditions.

\subsection{Surface Energies}

[23] Conservative estimates of $\gamma_{s}^{e}=\mathcal{G}_{0} / 2$ were made by recording the lowest measured $\mathcal{G}$ at which crack growth was observed, which gives upper bounds for the true values of $\mathcal{G}_{0}$. With increasing water concentration, we obtained surface energies of $0.32,0.30,0.23,0.12,0.14$ and $0.15 \mathrm{~J} / \mathrm{m}^{2}$ (Table 1 and Figure 8).

\subsection{Crack Surface Topography}

[24] Figure 9 shows the crack surface topography for one of the experiments $\left(c_{\mathrm{w}}=0.5\right)$. The crack surface morphologies are typical for cleavage surfaces, with steps repeating down to the nanometer scale. All of the crack surfaces show steps which are orthogonal to the crack front. This type of step is expected to form on cleavage surfaces due to crack interaction with screw dislocations [Gilman, 1959]. The 

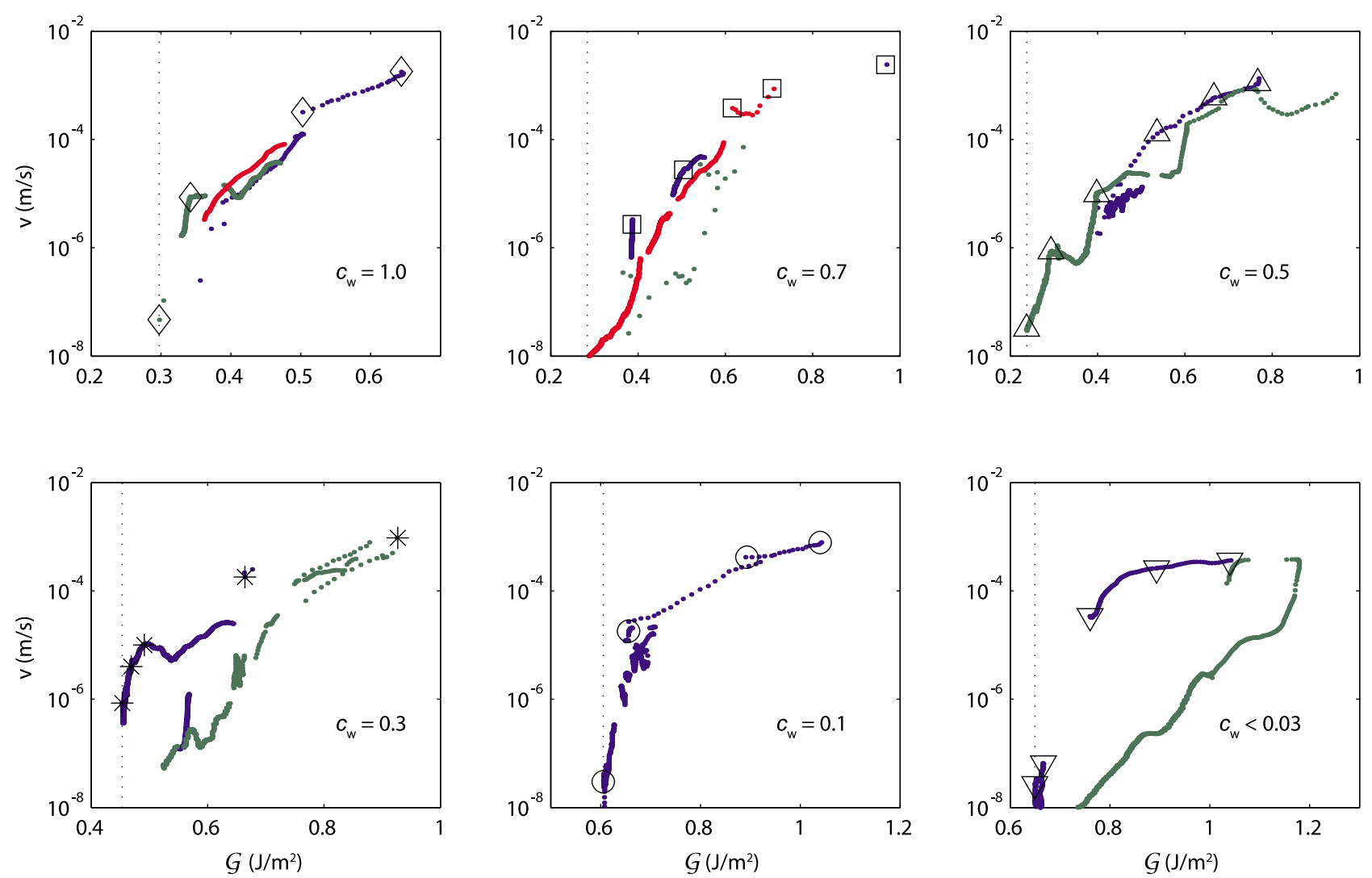

Figure 7. Results from the crack experiments. Each panel corresponds to a different water concentration, $c_{\mathrm{w}}$. Data points from all experiments are included and are shown in different colors. $\mathcal{G}_{0}$ is shown as a vertical dotted line. Open black symbols denote high-velocity points that we have extracted and shown in the collapse in Figure 10. Note that the velocity scale is the same for all frames, while the $\mathcal{G}$ scale varies.

ratio of vertical to horizontal displacement is $10^{-4}$ which means that the direct contribution of fracture steps normal to the fracture direction is negligible. One may also observe in Figure 9 that the surface step direction and step density does not seem to correlate with the crack velocity.

\section{Discussion}

\subsection{Surface Energy of Calcite}

[25] The measured surface energy of calcite (Figure 8) decreases with water concentration, and the value of $\gamma_{s}^{e}$ is more than doubled when the water concentration goes from 1 to close to 0 . This is consistent with the ratio between the dielectric constants and air/fluid surface tensions of glycol and water [Lide, 2008], which are 0.5.

[26] In Figure 8 we have compared our surface energy measurements with the molecular dynamics simulation data of de Leeuw and Parker [1997] for surface energies of the calcite cleavage plane at varying degrees of water coverage, from vacuum to one full monolayer at zero Kelvin. Our data and the data of de Leeuw and Parker [1997] show similar trends, suggesting that water causes an almost linear decrease in surface energy. Our data extend the already noticed discrepancy between results from experiments and modeling. For wet calcite surfaces, we would have expected experimental results to be higher than the numerical values, because the experimental calcite surfaces always contain steps. The lower experimental values for wet surfaces therefore strongly suggest that there are relaxation effects at water covered calcite surfaces that are not taken into account by current atomistic models. For the dry surfaces, we can explain the lower experimental values by considering that there is always some residual water adsorbed on the calcite surfaces, and in our case "dry" surfaces are covered with glycol, which also lowers the surface energy from the vacuum state.

[27] The trend in our data is consistent with the decrease in hydrostatic yield stress with increasing water activity that was found by Risnes et al. [2005] for chalk saturated with water-glycol mixtures. Our findings therefore support Risnes' hypothesis that the principal mechanism causing the water weakening effect is related to decreased cohesion of the chalk grains.

\subsection{Crack Velocity Model}

[28] Subcritical crack growth in region I (see Figure 1) can be understood as a thermally activated process as described by equation (1). Figure 10 shows a fit of this model to our data. Assuming a characteristic spacing of $a_{0}=5 \AA$ Stipp and Hochella, 1991], we get good agreement with our data with the parameters for the activation area $\alpha=2.9 \times$ $10^{-20} \mathrm{~m}^{2}$ and the quiescent energy barrier $\Delta F=7.6 \times 10^{-20} \mathrm{~J}$. 
Table 1. Comparison of Surface Energies $\gamma_{s}^{e}$ of Dry and Fully Hydrated Calcite Surfaces Found Experimentally and Numerically for the $\{10 \overline{1} 4\}$ Surface as Reported in Literature

\begin{tabular}{|c|c|c|}
\hline Reference & $\gamma_{s}^{e}, \operatorname{Dry}\left(\mathrm{J} / \mathrm{m}^{2}\right)$ & $\gamma_{s}^{e}$, Wet $\left(\mathrm{J} / \mathrm{m}^{2}\right)$ \\
\hline \multicolumn{3}{|c|}{ Experimental Results $^{\mathrm{a}}$} \\
\hline This study & 0.32 & 0.15 \\
\hline Donnet et al. [2005] & & $0.046 \pm 0.007$ \\
\hline Donnet et al. [2005] & & $0.135 \pm 0.029$ \\
\hline Donnet et al. [2009] & & $0.039-0.164$ \\
\hline Gilman [1960] & 0.23 & \\
\hline Santhanam and Gupta [1968] & $0.347 \pm 0.045$ & \\
\hline \multicolumn{3}{|c|}{ Simulation Results } \\
\hline de Leeuw and Parker [1997] & 0.60 & 0.30 \\
\hline de Leeuw et al. [1998] & 0.59 & 0.17 \\
\hline de Leeuw and Cooper [2004] & 0.59 & 0.33 \\
\hline Wright et al. [2001] & 0.32 & 0.23 \\
\hline Kerisit et al. [2003] & 0.59 & 0.21 \\
\hline Kvamme et al. [2009] & 0.86 & 0.29 \\
\hline
\end{tabular}

${ }^{\text {a }}$ The measurements of Donnet et al. [2005] are from precipitation studies, while those of Gilman [1960] and Santhanam and Gupta [1968] are from fracture experiments.

This can be compared with the results of Wan et al. [1990a] for mica, which are $\alpha=1.5 \times 10^{-19} \mathrm{~m}^{2}$ and $\Delta F=7.1 \times$ $10^{-20} \mathrm{~J}$ with $a_{0}=4.6 \AA$. There is no indication of a systematic change of fitting parameters with water concentration. This indicates that a single mechanism, independent of water concentration, controls subcritical crack growth in calcite at the velocities we have studied. The exact physical mechanism controlling subcritical crack growth in calcite cannot be indentified from these measurements. The activation areas correspond to length scales of $1.4 \AA$ for calcite and $3.9 \AA$ for mica, which are both on the order of the atomic spacing. This supports the notion of atomically sharp cleavage cracks in brittle crystals.

[29] Our findings and those of Dunning et al. [1994] indicate that the low-velocity plateau described by Henry et al. [1977] and Atkinson [1984] does not exist in single calcite crystals. If it is a real effect, it must be caused by some property of polycrystalline rocks rather than processes in calcite cleavage. This could be related to processes on the grain boundaries, or to effects related to plasticity in grains which are oriented favorably for twinning.

\subsection{Origin of Variable Crack Velocity Data}

[30] Our measured $v(\mathcal{G})$ curves (Figure 7) are highly variable, in particular at low $c_{\mathrm{w}}$ and high $\mathcal{G}$. A number of other studies have found subcritical crack growth to be more erratic with decreasing water concentration. For metals, this has been explained by plasticity in the form of local stickslip [Briggs et al., 1981] while in the case of mica, it has been attributed to surface charge effects [Deryagin and Metsik, 1960; Wan et al., 1990b]. In calcite, it has been shown experimentally that twins readily develop at the crack tip at room temperature. This process is reversible up to a certain stress threshold, but it is still a dissipative, nonelastic process [Bowden and Cooper, 1962]. It is therefore likely that there are stress-dependent, intermittent, dissipative

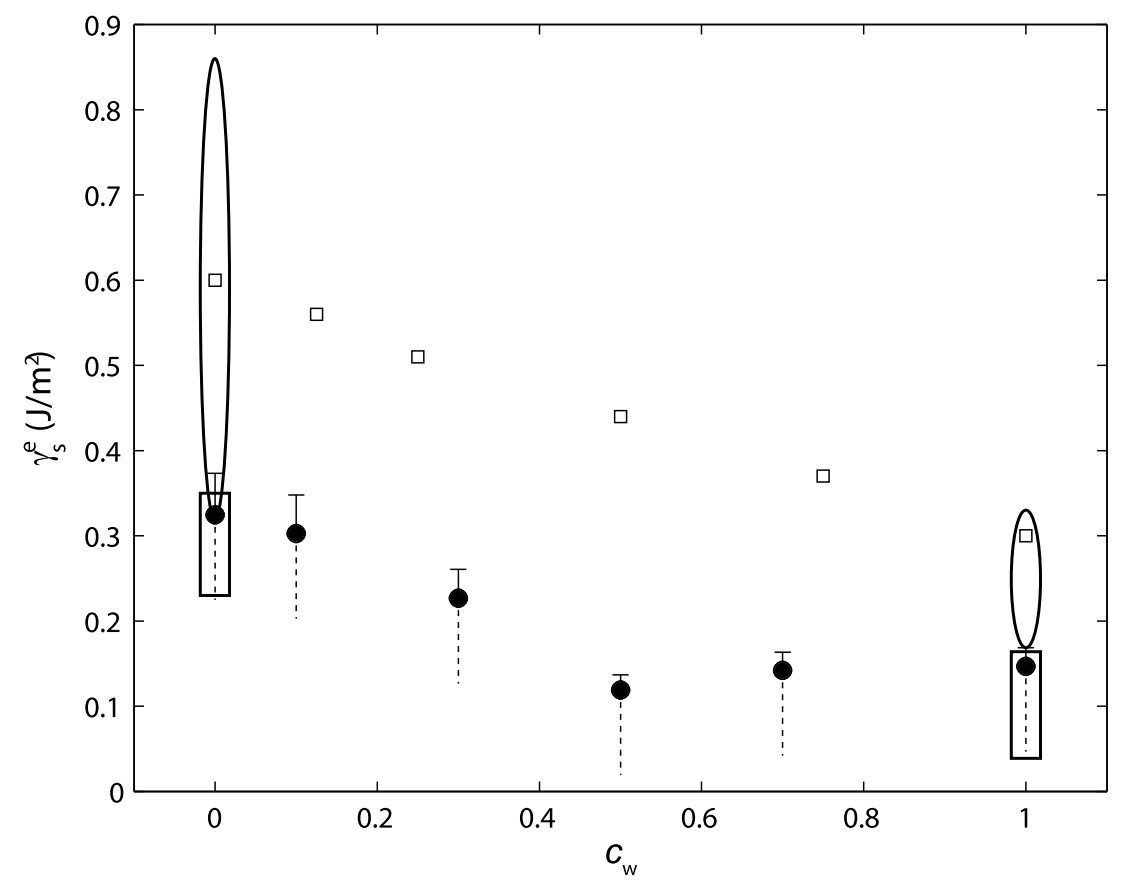

Figure 8. Surface energies $\gamma_{s}^{e}$ of calcite as a function of water concentration (mole fraction or surface cover fraction). Filled circles are estimates from experiments on calcite (this study). The measured $\gamma_{s}^{e}$ are plotted with asymmetric error bars: the upper shows the experimental uncertainty, while the lower is undefined, indicating that our values represent upper bounds. Open squares are surface energies for calcite surfaces with partial coverage of water, calculated by atomistic simulations by de Leeuw and Parker [1997]. Ellipses show the range of numerical results from literature for dry and wet calcite surfaces, while rectangles show the range of experimental results from literature (see Table 1). 

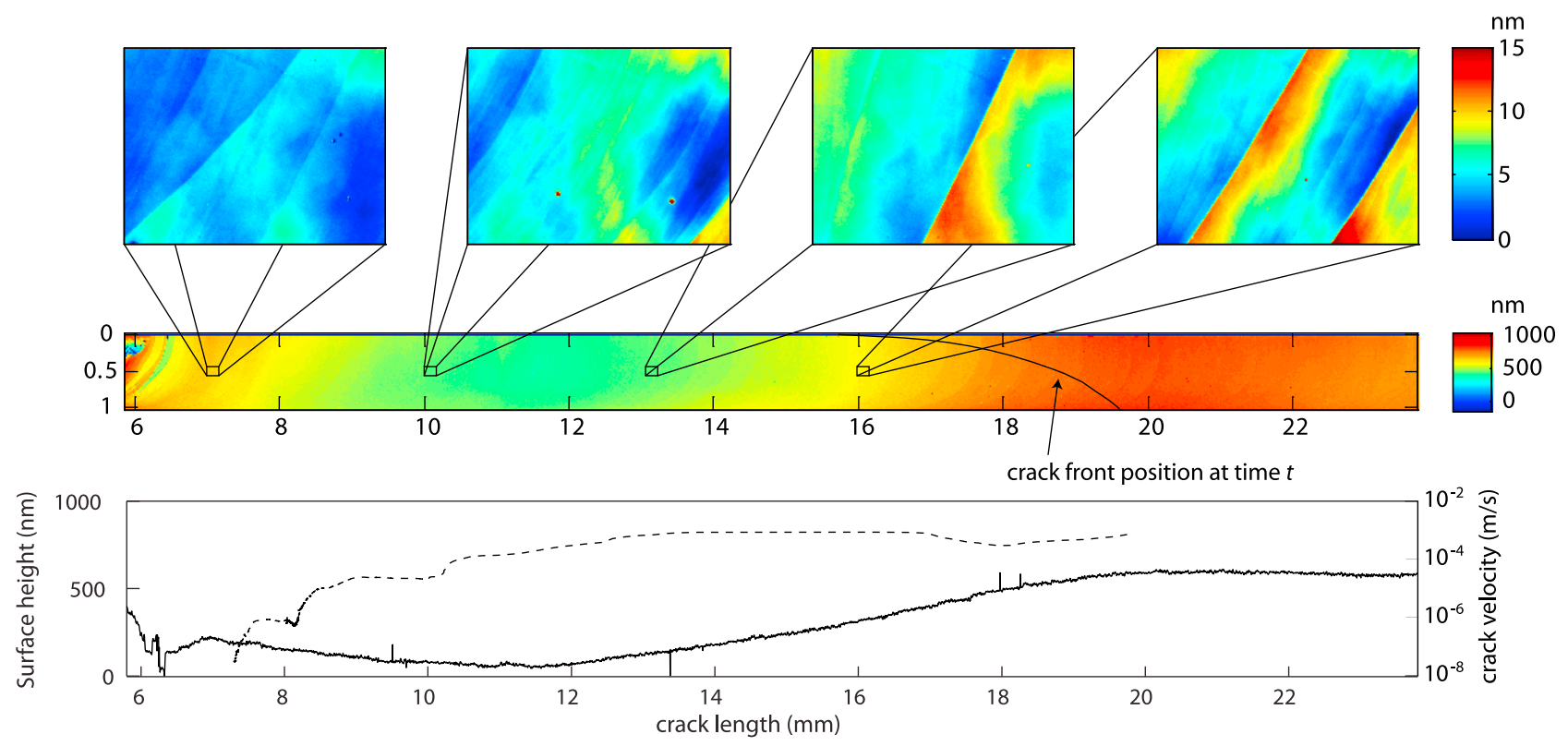

Figure 9. Crack surface topography of one of the 50\% water experiments (plotted in green in Figure 7) together with the surface height along the middle of the sample (solid line) and crack velocity (dashed line) as a function of crack length. No correlation was found between the variations in velocity and crack surface topography. Some $120 \times 90 \mu \mathrm{m}$ high-resolution surface images are also included, showing typical cleavage surface steps down to the crystal unit step height.

processes occurring at the crack tip in calcite which do not leave a visible trace on the crack surfaces.

[31] Variations may also be due to effects of crystallographic orientation. On the small scale, some left-right wandering around the cleavage plane is observed (Figure 9), which would result in variation of the ratio of type 1 to type 2 cleavage cracks (see Figure 4). Since type 1 cracks are expected to more energetically favorable, this may have an effect on the observed crack velocity. Slight sample misalignment may cause these effects to become more

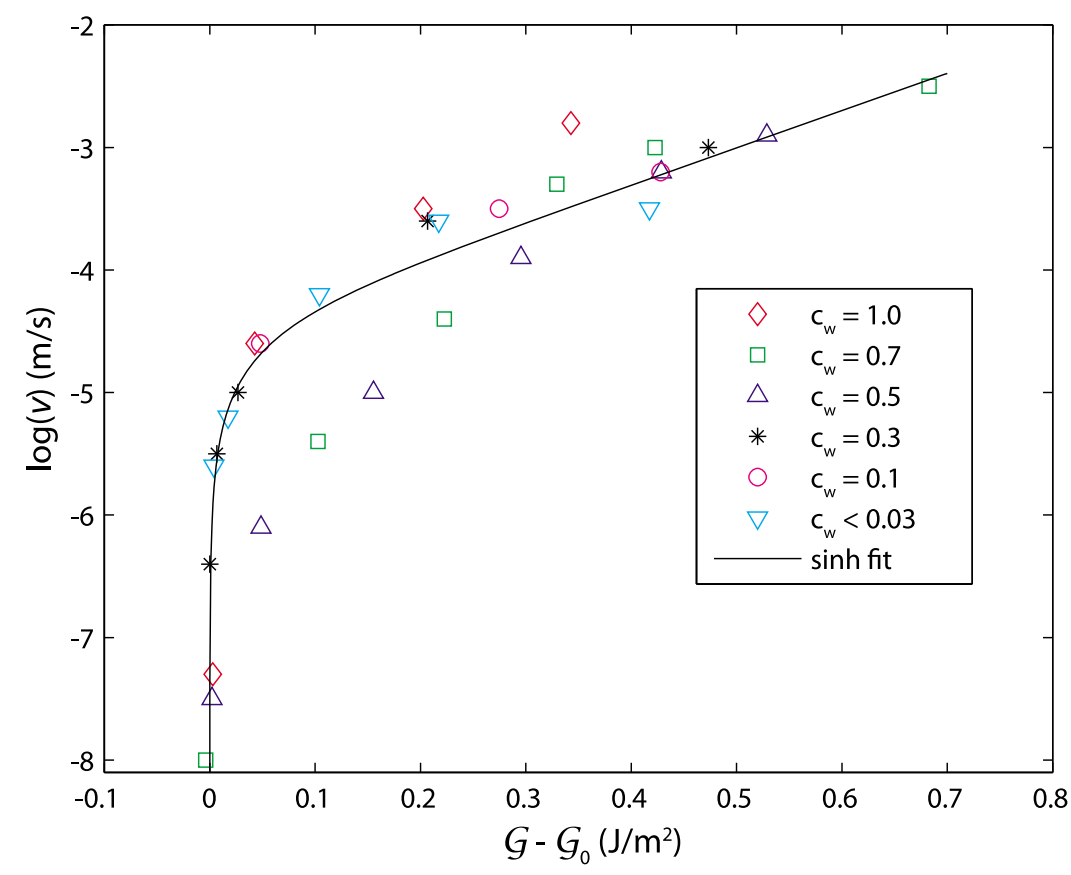

Figure 10. High-velocity points shown in Figure 7, plotted as a function of $\mathcal{G}-\mathcal{G}_{0}$. The line shows a fit to the model of Wan et al. [1990a] (equation (1)) for region I subcritical crack propagation, with fitting parameters $\alpha=2.9 \times 10^{-20} \mathrm{~m}^{2}$ and $\Delta F=7.6 \times 10^{-20} \mathrm{~J}$. 
dominant. More research is needed in order to fully understand the origin of the crack velocity variations.

\section{Conclusion}

[32] Double torsion experiments on subcritical cracking in calcite represent an alternative method for measuring the effect of chemical environment on the surface energy of calcite. Our results for different water concentrations are consistent with previous experimental results, and are consistently lower than results from numerical simulations. The discrepancy points to relaxation effects that are not properly accounted for in the simulations.

[33] The observed large variations in crack velocity at a given energy release rate may be caused by intermittent plastic processes in calcite, or by some other unidentified process. The highest measured velocities at any given $\mathcal{G}$, which are taken to represent the truly brittle behavior, can be well described using a reaction rate model with parameters similar to those for mica. These findings support the notion of an atomically sharp cleavage crack. There is no indication that the mechanism of subcritical crack growth in calcite is dependent on the water concentration.

[34] Subcritical crack measurements such as those presented here represent an opportunity for measuring the surface energies of different minerals in a wide range of chemical environments. Improved experimental control and higher optical resolution could yield better constrained estimates of $\mathcal{G}_{0}$. These experimental results would be highly valuable for the validation of numerical simulations. More knowledge on the surface energies of different minerals could also provide us with new insight on how fluids move and reactions take place in a heterogeneous rock.

[35] Acknowledgments. We thank Paul Meakin and Francois Renard for helpful comments to the manuscript. Comments made by the two reviewers significantly improved the paper. This study was supported by a Center of Excellence grant from the Norwegian Research Council to the Physics of Geological Processes (PGP) Center.

\section{References}

Agosta, F., M. Prasad, and A. Aydin (2007), Physical properties of carbonate fault rocks, Fucino Basin (central Italy): Implications for fault seal in platform carbonates, Geofluids, 7(1), 19-32.

Alcantar, N., J. Israelachvili, and J. Boles (2003), Forces and ionic transport between mica surfaces: Implications for pressure solution, Geochim. Cosmochim. Acta, 67(7), 1289-1304.

Anzalone, A., J. Boles, G. Greene, K. Young, J. Israelachvili, and N. Alcantar (2006), Confined fluids and their role in pressure solution, Chem. Geol., 230(3-4), 220-231.

Atkinson, B. K. (1984), Subcritical crack growth in geological materials, J. Geophys. Res., 89(B6), 4077-4114.

Bowden, F. P., and R. E. Cooper (1962), Velocity of twin propagation in crystals, Nature, 195(4846), 1091-1092.

Briggs, A., R. Airey, and B. C. Edwards (1981), A double torsion fracture mechanics and auger electron spectroscopy approach to the study of stress corrosion cracking in low alloy steels, J. Mater. Sci., 16(1), 125-140.

Chen, C. C., C. C. Lin, L. G. Liu, S. V. Sinogeikin, and J. D. Bass (2001), Elasticity of single-crystal calcite and rhodochrosite by brillouin spectroscopy, Am. Mineral., 86(11-12), 1525-1529.

Croizé, D., K. Bjørlykke, J. Jahren, and F. Renard (2010), Experimental mechanical and chemical compaction of carbonate sand, J. Geophys. Res., 115, B11204, doi:10.1029/2010JB007697.

de Gennes, P.-G. (2003), Capillarity and Wetting Phenomena: Drops, Bubbles, Pearls, Waves, Springer, New York. de Leeuw, N. H., and T. G. Cooper (2004), A computer modeling study of the inhibiting effect of organic adsorbates on calcite crystal growth, Cryst. Growth Des., 4(1), 123-133.

de Leeuw, N. H., and S. C. Parker (1997), Atomistic simulation of the effect of molecular adsorption of water on the surface structure and energies of calcite surfaces, J. Chem. Soc. Faraday Trans., 93(3), 467-475.

de Leeuw, N. H., S. C. Parker, and K. H. Rao (1998), Modeling the competitive adsorption of methanoic acid on calcite and fluorite surfaces, Langmuir, 14, 5900-5906.

Deryagin, B. V., and M. S. Metsik (1960), Role of electrical forces in the process of splitting mica along cleavage planes, Sov. Phys. Solid State, 1, 1393-1399.

Donnet, M., P. Bowen, N. Jongen, J. Lemaître, and H. Hofmann (2005), Use of seeds to control precipitation of calcium carbonate and determination of seed nature, Langmuir, 21(1), 100-108.

Donnet, M., P. Bowen, and J. Lemaître (2009), A thermodynamic solution model for calcium carbonate: Towards an understanding of multiequilibria precipitation pathways, J. Colloid Interface Sci., 340(2), $218-224$

Dunning, J., B. Douglas, M. Miller, and S. McDonald (1994), The role of the chemical environment in frictional deformation: Stress corrosion cracking and comminution, Pure Appl. Geophys., 143(1-3), 151-178.

Espinosa-Marzal, R. M., and G. W. Scherer (2010), Advances in understanding damage by salt crystallization, Acc. Chem. Res., 43(6), 897-905.

Evans, A. G. (1972), Method for evaluating time-dependent failure characteristics of brittle materials - and its application to polycrystalline alumina, J. Mater. Sci., 7(10), 1137-1146.

Fredrich, J. T., and B. Evans (1989), Micromechanics of the brittle to plastic transition in Carrara marble, J. Geophys. Res., 94(B4), 4129-4145.

Gilman, J. J. (1959), Cleavage, ductility and tenacity in crystals, in Fracture, edited by B. L. Averbach et al., pp. 193-222, John Wiley, New York.

Gilman, J. J. (1960), Direct measurement of the surface energies of crystals, J. Appl. Phys., 31(12), 2208-2218.

Hamilton, A., V. Koutsos, and C. Hall (2010), Direct measurement of saltmineral repulsion using atomic force microscopy, Chem. Commun., 46(29), 5235-5237.

Heap, M. J., P. Baud, and P. G. Meredith (2009), Influence of temperature on brittle creep in sandstones, Geophys. Res. Lett., 36, L19305, doi:10.1029/2009GL039373.

Helmstetter, A., and B. E. Shaw (2009), Afterslip and aftershocks in the rateand-state friction law, J. Geophys. Res., 114, B01308, doi:10.1029/ 2007JB005077

Henry, J., J. Paquet, and J. Tancrez (1977), Experimental study of crack propagation in calcite rocks, Int. J. Mech. Min. Sci. Geomech. Abstr., 14, 85-91.

Kerisit, S., S. C. Parker, and J. H. Harding (2003), Atomistic simulation of the dissociative adsorption of water on calcite surfaces, J. Phys. Chem. B, 107(31), 7676-7682.

Kvamme, B., T. Kuznetsova, and D. Uppstad (2009), Modelling excess surface energy in dry and wetted calcite systems, J. Math. Chem., 46, 756-762.

Lawn, B. (1993), Fracture of Brittle Solids, 2nd ed., Cambridge Univ. Press, Cambridge, U. K.

Lide, D. R. (Ed.) (2008), CRC Handbook of Chemistry and Physics: A Ready-Reference Book of Chemical and Physical Data, 89th ed., CRC Press, Boca Raton, Fla.

Maugis, D. (1985), Review: Subcritical crack growth, surface energy, fracture toughness, stick-slip and embrittlement, J. Mater. Sci., 20 3041-3073.

Miller, S. A., C. Collettini, L. Chiaraluce, M. Cocco, M. Barchi, and B. J. P. Kaus (2004), Aftershocks driven by a high-pressure $\mathrm{CO}_{2}$ source at depth, Nature, 427(6976), 724-727.

Olagnon, C., J. Chevalier, and V. Pauchard (2006), Global description of crack propagation in ceramics, J. Eur. Ceram. Soc., 26(15), 3051-3059.

Risnes, R., M. Madland, M. Hole, and N. Kwabiah (2005), Water weakening of chalk - mechanical effects of water-glycol mixtures, J. Petrol. Sci. Eng., 48, 21-36.

Sandengen, K. (2006), Prediction of mineral scale formation in wet gas condensate pipelines and in MEG (Mono Ethylene Glycol) regeneration plants, Ph.D. thesis, Norw. Univ. of Sci. and Technol., Trondheim, Norway.

Santhanam, A. T., and Y. P. Gupta (1968), Cleavage surface energy of calcite, Int. J. Mech. Min. Sci. Geomech. Abstr., 5(3), 253-259.

Scholz, C. H. (2002), The Mechanics of Earthquakes and Faulting, 2nd ed. Cambridge Univ. Press, Cambridge, U. K.

Schubnel, A., E. Walker, B. D. Thompson, J. Fortin, Y. Gueguen, and R. P. Young (2006), Transient creep, aseismic damage and slow failure in Carrara marble deformed across the brittle-ductile transition, Geophys. Res. Lett., 33, L17301, doi:10.1029/2006GL026619. 
Shyam, A., and E. Lara-Curzio (2006), The double-torsion testing technique for determination of fracture toughness and slow crack growth behavior of materials: A review, J. Mater. Sci., 41(13), 4093-4104.

Stipp, S. L., and M. F. Hochella (1991), Structure and bonding environments at the calcite surface as observed with X-ray photoelectronspectroscopy (XPS) and low-energy electron-diffraction (LEED), Geochim. Cosmochim. Acta, 55(6), 1723-1736.

Turner, F. J., D. T. Griggs, and H. Heard (1954), Experimental deformation of calcite crystals, Geol. Soc. Am. Bull., 65(9), 883-934.

Vanel, L., S. Ciliberto, P. P. Cortet, and S. Santucci (2009), Timedependent rupture and slow crack growth: Elastic and viscoplastic dynamics, J. Phys. D Appl. Phys., 42, 214007, doi:10.1088/0022$3727 / 42 / 21 / 214007$.

Wan, K.-T., S. Lathabai, and B. R. Lawn (1990a), Crack velocity functions and thresholds in brittle solids, J. Eur. Ceram. Soc., 6, 259-268.
Wan, K.-T., N. Aimard, S. Lathabai, R. G. Horn, and B. R. Lawn (1990b), Interfacial energy states of moisture-exposed cracks in mica, J. Mater. Res., 5, 172-182.

Wright, K., R. T. Cygan, and B. Slater (2001), Structure of the (1014) surfaces of calcite, dolomite and magnesite under wet and dry conditions, Phys. Chem. Chem. Phys., 3(5), 839-844.

J. Bisschop, Institute for Building Materials, ETH Hönggerberg, HIF E 13.2, Schafmattstr. 6, CH-8093 Zürich, Switzerland. (jbisschop@ethz.ch)

D. K. Dysthe and A. Røyne, Physics of Geological Processes, University of Oslo, PO Box 1048 Blindern, Oslo N-0316, Norway. (d.k.dysthe@fys. uio.no; anja.royne@fys.uio.no) 